\title{
Use of Chebychev Polynomials in Thin Film Computations
}

\author{
Klaus D. Mielenz
}

(August 17, 1959)

\begin{abstract}
From Herpin's expression for the $m$ th power of a multilayer matrix, very simple closed formulas are derived for the matrices and optical constants of any multilayer with a periodic structure.

According to Epstein's theorem, any symmetrical multilayer is equivalent to a fictitious monolayer. A simple expression for the equivalent index and thickness of this monolayer is deduced for the case of a periodic and symmetrical sequence of equally thick films.

As compared to any other method of numerical computation, the suggested formulation provides a considerable saving of time and work. In a numerical example, this saving amounts to about 80 percent.
\end{abstract}

\section{Fundamental Relationships $[1,2]^{1}$}

The electromagnetic field at the plane of entry to a multilayer as in figure 1a is determined by

$$
\begin{aligned}
\left(\begin{array}{l}
E_{0} \\
H_{0}
\end{array}\right)=\mathfrak{A}_{1} \mathfrak{A}_{2} \ldots \mathfrak{U}_{N}\left(\begin{array}{c}
1 \\
M_{s}
\end{array}\right), \\
\mathfrak{U}_{\nu}=\left(\begin{array}{cc}
\cos B_{\nu} & \frac{i}{M_{\nu}} \sin B_{\nu} \\
i M_{\nu} \sin B_{\nu} & \cos B_{\nu}
\end{array}\right),\left|\mathfrak{U}_{\nu}\right|=1,
\end{aligned}
$$

where

$$
M_{\nu}=\frac{m_{\nu}}{\mu_{\nu}}=\frac{n_{\nu}\left(1-i k_{\nu}\right)}{\mu_{\nu}}, \quad B_{\nu}=\frac{2 \pi}{\lambda} m_{\nu} d_{\nu} \cos \phi_{\nu}
$$

are the complex index and the optical thickness in phase units of the $\nu$ th layer $\left(n_{\nu}=\right.$ refractive index, $\dot{c}_{v}=$ absorption coefficient, $\mu_{\nu}=$ permeability, $d_{\nu}=$ hysical thickness, $\lambda=$ wavelength, and $\phi_{\nu}=$ angle f incidence).

The amplitude transmission and reflection coefficients of the multilayer are

$$
T=\frac{2 M_{0} \sqrt{M_{s}+M_{s}^{*}}}{\sqrt{M_{0}+M_{0}^{*} C}}, \quad R=\frac{D}{C}
$$

with

$$
C=M_{0} E_{0}+H_{0}, \quad D=M_{0} E_{0}-H_{0} .
$$

Various methods for computing $R$ and $T$ have been suggested in the literature. They all have the disadvantage of being based upon recurrence relations that make it necessary to calculate the desired quantities in a cumbersome stepwise manner. Although such a procedure seems to be inevitable in

\footnotetext{
1 Figures in brackets indicate the literature references at the end of this paper.
}
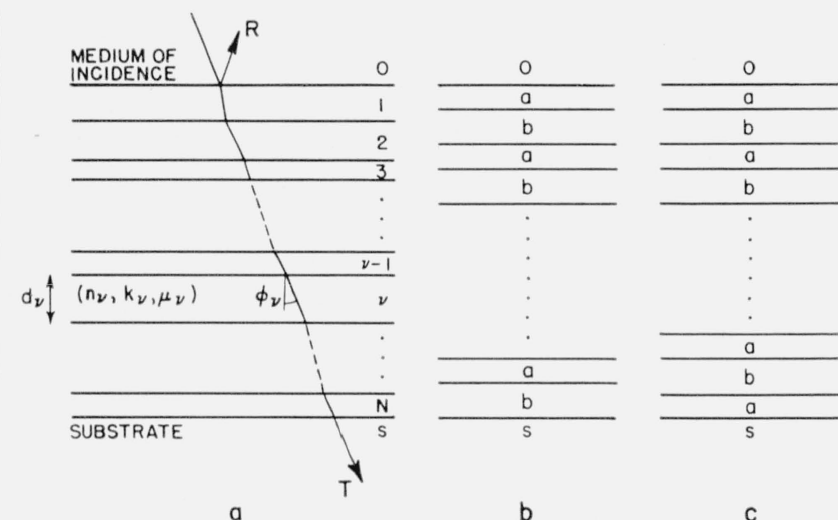

Figure 1: Optical multilayers.

(a) General case and denotations, (b) periodic, and (c) periodic-symmetrical multilayer. (In figures $1 \mathrm{~b}$ and $\mathrm{c}$, the individual layers $a$ and $b$ do not necessarily
represent single films.)

general, a much simpler approach is possible with "periodic" and "periodic-symmetrical" multilayers as represented in figures $1 \mathrm{~b}$ and $\mathrm{c}$. Since such multilayers are of considerable importance in thin film work, the computation method developed hereafter is of great practical significance.

\section{2. "Periodic" Multilayers}

A multilayer in which the same sequence of films is repeated twice or more often is a "periodic" multilayer. According to Herpin's theorem [3] any multilayer, and therefore the fundamental period of layers as well, may be expressed as a fictitious bilayer the matrix of which we shall call $\mathfrak{A}_{a} \mathfrak{H}_{b}$. If the period occurs $m$ times the matrix eq (1) reads

$$
\left(\begin{array}{c}
E_{0} \\
H_{0}
\end{array}\right)=\left(\mathfrak{A}_{a} \mathfrak{A}_{b}\right)^{m}\left(\begin{array}{c}
1 \\
M_{s}
\end{array}\right) \text {. }
$$




\section{3. "Periodic-Symmetrical" Multilayers}

A multilayer for which the indices and thicknesses are the same as encountered from either side,

$$
\left(\begin{array}{l}
E_{0} \\
H_{0}
\end{array}\right)=\mathfrak{A}_{\alpha} \mathfrak{H}_{\alpha-1} \ldots \mathfrak{A}_{2} \mathfrak{H}_{1} \mathfrak{H}_{2} \ldots \mathfrak{A}_{\alpha-1} \mathfrak{H}_{\alpha}\left(\begin{array}{c}
1 \\
M_{s}
\end{array}\right),
$$

is a "symmetrical" multilayer. It can be replaced by a fictitious monolayer (Epstein [4]).

If such a multilayer consists of $[m+(1 / 2)]$ times a fundamental period,

$$
\left(\begin{array}{l}
E_{0} \\
H_{0}
\end{array}\right)=\left(\mathfrak{A}_{a} \mathfrak{U}_{b}\right)^{m} \mathfrak{A}_{a}\left(\begin{array}{c}
1 \\
M_{s}
\end{array}\right),
$$

it is a "periodic-symmetrical" multilayer.

\section{The mth Power of a Matrix of Unity Determinant}

A simple closed expression for $\left(\mathfrak{A}_{a} \mathfrak{U}_{b}\right)^{\mathrm{m}}$ is the key to eqs (5) and (6). Herpin [5] has shown that the powers of a four-element matrix can be expressed by Lucas polynomials, and Abelès $[6,1]$ has observed that these are reduced to Chebycher polynomials if the basic matrix is of unity determinant (which is the case for multilayer matrices). This principle, however, has not been developed further since.

Let $\mathfrak{A}$ be any four element matrix of determinant unity and write

$\mathfrak{U}=\left(\begin{array}{ll}a_{11} & a_{12} \\ a_{21} & a_{22}\end{array}\right)=a \sigma_{0}+b \sigma_{1}+c \sigma_{2}$

$$
+d \sigma_{3}, \quad a^{2}-\left(b^{2}+c^{2}+d^{2}\right)=1,
$$

with

$\sigma_{0}=\left(\begin{array}{ll}1 & 0 \\ 0 & 1\end{array}\right), \quad \sigma_{1}=\left(\begin{array}{ll}0 & 1 \\ 1 & 0\end{array}\right)$,

$$
\sigma_{2}=\left(\begin{array}{rr}
0 & -i \\
i & 0
\end{array}\right), \quad \sigma_{3}=\left(\begin{array}{rr}
1 & 0 \\
0 & -1
\end{array}\right)
$$

(Pauli spin matrices). Then, we obtain

$$
\mathfrak{U}^{2}=2 a \mathfrak{U}-\sigma_{0} .
$$

Following Herpin, we set

$$
\mathfrak{U}^{m}=S_{m-1} \mathfrak{A}-S_{m-2} \sigma_{0},
$$

whence we arrive at

$$
\begin{aligned}
\mathfrak{H}^{m+1} & =S_{m} \mathfrak{Y}-S_{m-1} \sigma_{0}=\mathfrak{U}^{m} \mathfrak{Y}=S_{m-1} \mathfrak{H}^{2}-S_{m-2} \mathfrak{A} \\
& =2 a S_{m-1} \mathfrak{A}-S_{m-1} \sigma_{0}-S_{m-2} \mathfrak{A} .
\end{aligned}
$$

Putting

$$
2 a=a_{11}+a_{22}=X,
$$

we find by comparison of coefficients, as recurrence formula for the $S_{m}$ 's,

$$
S_{m}(X)=X S_{m-1}(X)-S_{m-2}(X) .
$$

Equation (9) yields the initial values,

$$
S_{0}(X)=1, S_{1}(X)=X,
$$

and then (12) leads to

$$
S_{m}(X)=\sum_{\mu=0}^{[m / 2]}(-1)^{\mu}\left(\begin{array}{c}
m \\
\mu
\end{array}\right) X^{m-2 \mu},
$$

where $[\mathrm{m} / 2]$ denotes the largest integer contained in $\mathrm{m} / 2$, e.g., $[5 / 2]=2$. Explicitly, we have

$$
\begin{aligned}
& S_{0}=1, \\
& S_{1}=X, \\
& S_{2}=X^{2}-1, \\
& S_{3}=X^{3}-2 X, \\
& S_{4}=X^{4}-3 X^{2}+1, \\
& S_{5}=X^{5}-4 X^{3}+3 X, \\
& S_{6}=X^{6}-5 X^{4}+6 X^{2}-1, \\
& S_{7}=X^{7}-6 X^{5}+10 X^{3}-4 X, \\
& S_{8}=X^{8}-7 X^{6}+15 X^{4}-10 X^{2}+1, \\
& \quad \text { etc. }
\end{aligned}
$$

The $S_{m}$ 's defined by these equations are the Chebycher polynomials of the second kind [7],

$$
S_{m}(X)=\frac{\sin (m+1) \Theta}{\sin \theta}, \quad X=2 \cos \theta,
$$

or

$$
S_{m}(X)=\frac{\sinh (m+1) \Phi}{\sinh \Phi}, \quad X=2 \cosh \Phi
$$

Another form is

$$
\begin{aligned}
& S_{m}(X)=\frac{1}{2^{m+1} \sqrt{X^{2}-4}}\left[\left(X+\sqrt{X^{2}-4}\right)^{m+1}\right. \\
&\left.-\left(X-\sqrt{X^{2}-4}\right)^{m+1}\right] .
\end{aligned}
$$

For real arguments, $X=x$, these polynomials are also real (even though this is not obvious in eq (16) for $|x|<2$ ). If, in eqs (15), $\theta$ and $\Phi$ are to be real for reasons of convenience, (15a) must be used for $|x| \leq 2$, and (15b) for $|x| \geq 2$.

The desired matrix $\left(\mathfrak{U}_{a} \mathfrak{A}_{b}\right)^{m}$ may now be obtained as follows: Form

$$
\mathfrak{A}_{a} \mathfrak{U}_{b}=\left(\begin{array}{ll}
a_{11} & a_{12} \\
a_{21} & a_{22}
\end{array}\right) \text { and } X=a_{11}+a_{22} .
$$

Then, find $S_{m-1}(X)$ and $S_{m-2}(X)$ and write

$$
\left(\mathfrak{H}_{a} \mathfrak{H}_{b}\right)^{m}=\left(\begin{array}{cc}
S_{m-1} a_{11}-S_{m-2} & S_{m-1} a_{12} \\
S_{m-2} a_{21} & S_{m-1} a_{22}-S_{m-2}
\end{array}\right) .
$$




\section{Application to Multilayers}

According to (5), (6), and (17), we have

$$
F_{P}=S_{m-1}(X) \cdot F_{a b}-S_{m-2}(X) \cdot F_{s},
$$

and

$$
F_{P S}=S_{m-1}(X) \cdot F_{a b a}-S_{m-2}(X) \cdot F_{a},
$$

where $F$ stands for either field strength, $E$ or $H$, and because of (4) for the quantities $C$ and $D$ as well. The subscripts $P$ and $P S$ refer to the whole periodic and periodic-symmetrical multilayers, respectively; the subscripts $s, a, a b$, and $a b a$ refer to the uncoated substrate, the bottom mono-, bi-, and trilayers, respectively.

Thus, any of these quantities can be expressed as a simple linear combination of the corresponding quantities of much simpler multilavers.

$\mathrm{By}$ forming the matrix product in (16), one finds the argument of the Chebychev polynomials in (18) and (19),

$$
X=2 \cos B_{a} \cos B_{b}-\frac{M_{a}^{2}+M_{b}^{2}}{M_{a} M_{b}} \sin B_{a} \sin B_{b} .
$$

\section{Layers of Equal Thickness}

The equations of section 5 are still further simplified if each film in the multilaver has the same optical thickness,

$$
B_{a}=B_{b}=B .
$$

Then, we have

$$
X=2-\frac{\left(M_{a}+M_{b}\right)^{2}}{M_{a} M_{b}} \sin ^{2} B
$$

While for the periodic multilaver the mathematical formulation itself is not simplified, a very simple formulation is obtained for the periodic-symmetrical multilayer:

With (21) and (22), the matrix of the trilayer (aba) can be shown to be

$$
\mathfrak{U}_{a} \mathfrak{U}_{b} \mathfrak{U}_{a}=X \mathfrak{A}_{a}-\mathfrak{H}_{b}^{-1},
$$

where

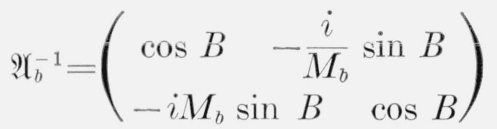

is the inverse of $\mathfrak{U}_{b}$. Then, (17) and (12) show that

$$
\left(\mathfrak{H}_{a} \mathfrak{Y}_{b}\right)^{m} \mathfrak{A}_{a}=S_{m}(X) \mathfrak{P}_{a}-S_{m-1}(X) \mathfrak{H}_{b}^{-1} .
$$

Because of (13), (23) is contained in (25) as the particular case $m=1$.

Thus we see that, if all films are equally thick, it is not necessary to express the periodic-symmetrical multilayer in terms of the bottom trilayer and the uncoated substrate, as in (19). Instead, it can very simply be expressed in terms of the two basic matrices $\mathfrak{U}_{a}$ and $\mathfrak{Y}_{b}$, without any need for multiplication of matrices whatsoever.

Epstein's theorem [4], according to which any symmetrical multilayer is equivalent to a fictitious monolayer, was already mentioned. The index $M_{m}$ and the thickness $B_{m}$ of the monolayer corresponding to a periodic-symmetrical multilayer with equally thick films may now be obtained as follows:

Consider (25) and write

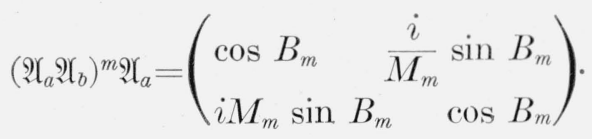

Then, comparison of coefficients yields, for the thickness $B_{m}$ and the index $M_{m}$ of the fictitious monolayer,

$$
\begin{aligned}
\cos B_{m}= & {\left[S_{m}(X)-S_{m-1}(X)\right] \cos B, } \\
M_{m}^{2}= & \frac{M_{a} S_{m}(X)+M_{b} S_{m-1}(X)}{\frac{1}{M_{a}} S_{m}(X)+\frac{1}{M_{b}} S_{m-1}(X)}
\end{aligned}
$$

(According to Epstein [4], the signs of $B_{m}$ and $M_{m}$ have to be chosen such that $B_{m} \rightarrow 0$ for $\lambda \rightarrow \infty$, and that always $\operatorname{Re}\left(M_{m}\right) \geq 0$.

\section{Dielectric Multilayers}

The above formulation constitutes a considerable simplification of practical computations.

An automatic computer can provide for itself the needed Chebycher polynomials by computing them according to eqs (14) or (16), regardless of whether $X$ is a real or a complex number.

For desk calculations, however, numerical values of Chebychev polynomials of complex arguments cannot be found except with rather complicated calculations. This leads us to the restriction that $X$ always should be a real number which will be true only if the multilayers are purely dielectric, and if the individual matrices $\mathfrak{A}_{a}$ and $\mathfrak{A}_{b}$ represent individual films. (The replacement of multilayers, even if purely dielectric, by fictitious mono- or bilayers may yield complex indices.) These assumptions lead to the important class of alternating dielectric lavers, for which we have

$$
M_{a}=m_{a}=n_{a}, \quad M_{b}=m_{b}=n_{b},
$$

and (for equally thick layers that are quarter wave films at a wavelength $\lambda_{0}$ )

$$
B_{a}=B_{b}=\beta=\frac{\pi}{2} \frac{\lambda_{0}}{\lambda}
$$

so that

$$
X=x=2-\frac{\left(n_{a}+n_{b}\right)^{2}}{n_{a} n_{b}} \sin ^{2} \beta
$$

is a real number. 
For such real arguments, the Chebychev polynomials may be found with the aid of:

: (a) Numerical tables: 12-decimal values of the first $12 S_{m}{ }^{\prime}$ s, for $0 \leq x \leq 2$ with intervals 0.001 in $x$, have been published by the National Bureau of Standards [7]. Jones and co-authors [8] have published similar but less complete tables. For negative arguments, find $S_{m}(|x|)$ and use the relation

$$
S_{m}(-x)=(-1)^{m} S_{m}(x) \text {. }
$$

(b) Equation (15) and tables of trigonometric or hyperbolic functions: If, for instance, $S_{4}(3.745)$ is looked for, one finds from the table that $3.745=2$ cosh 1.24. Hence $\Phi=1.24,5 \Phi=6.20$, and

$$
S_{4}(3.745)=\frac{\sinh 6.20}{\sinh 1.24}=\frac{246.37}{1.58311}=155.62 .
$$

(c) Direct computation: The $S_{m}$ 's may also be computed from eq (14) or (16). For small values of $m$ it is easier to use eqs (14), but for large $m^{\prime} \mathrm{s}$ (which, however, rarely occur in multilayer work) eq (16) is faster.

\section{A Practical Example}

Consider a high reflection multilayer consisting of 11 alternating zinc sulfide and magnesium fluoride films on glass,

$$
\begin{array}{cl}
n_{0}=1(\text { air }), & n_{a}=2.3(\mathrm{ZnS}), \\
n_{b}=1.38\left(\mathrm{MgF}_{2}\right), & n_{s}=1.52 \text { (glass). }
\end{array}
$$

Let all films be a quarter wave thick at $\lambda_{0}=5460.74 \mathrm{~A}$, and compute the amplitude reflection coefficient $R$ for $\lambda=4358.35$ A, i.e., for

$$
\begin{aligned}
\beta=\frac{\pi}{2} \frac{5460.74}{4358.35}=112^{\circ} 45.86^{\prime}, \\
\sin \beta=0.92211, \cos \beta=-0.38694 .
\end{aligned}
$$

Then we have according to (1), (24), and (25),

$$
\begin{aligned}
& \left(\begin{array}{l}
E_{0} \\
H_{0}
\end{array}\right)=\left[S_{5}(x)\left(\begin{array}{cc}
-0.38694 & 0.40092 i \\
2.12085 i & -0.38694
\end{array}\right)\right. \\
& \left.-S_{4}(x)\left(\begin{array}{ll}
-0.38694 & -0.66820 i \\
-1.27252 i & -0.38694
\end{array}\right)\right]\left(\begin{array}{l}
1 \\
1.52
\end{array}\right) .
\end{aligned}
$$

From (31), we obtain $x=-1.62789$, so that we may look up in reference [7]: $S_{5}(x)=0.94004, S_{4}(x)=$ 0.07257 .

Thence,

$$
\begin{array}{r}
\left(\begin{array}{l}
E_{0} \\
H_{0}
\end{array}\right)=\left[\begin{array}{rr}
-0.36374 & 0.37688 i \\
1.99363 i & -0.36374
\end{array}\right) \\
\left.+\left(\begin{array}{cc}
0.02808 & 0.04849 i \\
0.09235 i & 0.02808
\end{array}\right)\right]\left(\begin{array}{l}
1 \\
1.52
\end{array}\right) \\
=\left(\begin{array}{rr}
-0.33566 & 0.42537 i \\
2.08603 i & -0.33566
\end{array}\right)\left(\begin{array}{l}
1 \\
1.52
\end{array}\right) \\
=\left(\begin{array}{l}
-0.33566+0.64656 i \\
-0.51020+2.08603 i
\end{array}\right)
\end{array}
$$

Finally, (3) and (4) yield

$$
R=\frac{0.17454-1.43917 i}{-0.84586+2.73259 i}
$$

Starting with the given $n$ 's and with $\beta$, this result was obtained with 17 individual steps of multiplication or division and 9 steps of addition or subtraction. Four numerical values had to be looked up in tables.

In comparison hereto, it takes 96 multiplications, and 48 additions or subtractions, with 1 numerical value to be looked up, to arrive at the same result by means of the widely used recurrence method for admittances [9]. This may be estimated to be about five times as much work.

\section{References}

[1] F. Abelès, Ann. phys. (12) 5, 596, 706 (1950).

[2] R. W. Muchmore, J. Opt. Soc. Am. 38, 20 (1948).

[3] A. Herpin, Compt. rend. 2\%5, 182 (1947).

[4] L. I. Epstein, J. Opt. Soc. Am. 42, 806 (1952).

[5] A. Herpin, Compt. rend. 225, 17 (1947).

[6] F. Abelès, Compt. rend. 2\%6, 1808 (1948).

[7] NBS Applied Math. Series 9 (1952).

[8] C. W. Jones, et al., Proc. Roy. Soc. (Edinburgh) 62, 187 (1946).

[9] P. J. Leurgans, J. Opt. Soc. Am. 41, 714 (1951).

Washington, D.C.

(Paper 63A3-24) 


\section{Publications of the National Bureau of Standards}

\section{Selected Ábstracts}

Measurement of the aging of rubber vulcanizates, J. Mandel, F. L. Roth, M. N. Steel, and R. D. Stiehler, J. Research NBS 63C No. 2, 141 (1959).

A study of aging data in the literature and of measurements made at the National Bureau of Standards indicates that ultimate elongation is the best of the tensile properties for characterizing the deterioration of rubber vulcanizates during storage at various temperatures. Ultimate elongation decreases during aging for all types of rubber vulcanizates; whereas, tensile strength and modulus may increase, decrease, or remain essentially unchanged. The change in ultimate elongation over prolonged periods of storage cannot be expressed by a simple mathematical equation. However, during most of the useful storage life of a rubber vulcanizate, the elongation decreases approximately linearly with the square root of time. The data indicate that for some vulcanizates an estimate of storage life at room temperature can be made from measurements of ultimate elongation at two or more elevated temperatures.

Excitation mechanisms of the oxygen 5577 emission in the upper atmosphere, E. Tandberg-Hanssen and F. E. Roach, J. Research NBS 63D No. 3, 319 (1959).

Possible excitation mechanisms for the green 5577 emission are considered in the light of recent data on the dynamics of the upper atmosphere. Photochemical reactions as affected by mass motions as well as excitation directly due to the mass motions are analyzed.

\section{Method for measuring local electron density from an artifical satellite L. R. O. Storey, J. Research NBS 63D No. 3, 325 (1959).}

A method is proposed for measuring the electron density at known points in the outer ionosphere, by the use of vlf receiving equipment in an artificial satellite, in conjunction with a vlf transmitter on the ground. The transmitter would radiate continuous waves, which would be propagated through the ionosphere in the 'whistler' mode. The basis of the method is a measurement of the local wave admittance of the medium, by comparison of the signals received on an electric dipole and on a loop.

A further proposal is made for an integrated vlf satellite experiment, in which several different types of observation would be made simultaneously.

\section{Reflectors for a microwave Fabry-Perot interfer- ometer W. Culshaw, IRE Trans. on Microwave Theory and Techniques, Vol. MTTT-y, No. 2, 221 (1959).}

The advantages of microwave interferometers for wavelength and other measurements at millimeter wavelengths are indicated, and a microwave Fabry-Perot interferometer discussed in detail. Analogous to the cavity resonator, this requires reflectors of high reflectivity, small absorption, and adequate size. Stacked dielectric plates, and stacked planar or rod gratings are shown to be suitable forms of reflectors, and equations for the reflectivity, optimum spacing, and bandwidth of such structures are derived. A series of stacked metal plates with regularly spaced holes represents a good design of reflector for very small wavelengths. Fringes and wavelength measurements at $8-\mathrm{mm}$ wavelength are given for one design of interferometer, these being accurate to 1 in $10^{4}$ without any diffraction correction. For larger apertures and reflectors in terms of the wavelength, errors due to diffraction will decrease.
The nature, cause, and effect of the porosity in electrodeposits, III. Microscopic detection of porosity, F. Ogburn and D. W. Ernst, Plating 46, 831 (1959).

A new technique called "parallel sectioning" was used for investigating the porosity of nickel electrodeposits. Some of the coating is polished off in a layer parallel to the basis metal. After taking a photomicrograph of this area, another layer of metal is removed. This procedure is continued until the basis metal has been reached. It is possible to construct a three-dimensional model of the coating. Various pore types are listed and illustrative photomicrographs are given.

Mechanism of contraction in the muscle fiber-ATP system, L. Mandelkern, A. S. Posner, A. F. Diorio, and K. Laki, Proc. Nat. Acad. Sci. U.S. 45, No. 6, $814(1959)$.

The axial contraction that develops when glycerol-treated rabbit psoas muscle was immersed in ATP solutions of varying concentrations was measured. The length-concentration diagram has the characteristics typical of a cooperative phase transformation and the shrinkage can be attributed to melting of the initially axially oriented polypeptide chains. This conclusion was substantiated by wide angle $\mathrm{x}$-ray diffraction studies of the native and shrunken fibers which show the structural changes expected on melting. Thus, the same principles that govern the contractile processes in a wide variety of fibrous macromolecular systems are also operative in the muscle-ATP system.

Microwave spectrum of methyl germane, V. W. Laurie, J. Chem. Phys. 30, No. 5, 1210 (1959).

The $J=0 \rightarrow 1$ and $1 \rightarrow 2$ transitions of 28 isotopic species of $\mathrm{CH}_{3} \mathrm{GeH}_{3}$ have been measured. From the rotational constants obtained, the following structural parameters have been calculated: $r_{\mathrm{CH}}=1.083+0.005 \mathrm{~A}^{\circ}, r_{\mathrm{GeH}}=1.529+0.005 \mathrm{~A}^{\circ}$ $r_{\mathrm{CG}}=1.9453 \pm 0.0005 \mathrm{~A}^{\circ}, \Varangle \mathrm{HCH}=108^{\circ} 25^{\prime} \pm 30^{\prime}, \Varangle \mathrm{HGeH}$ $=109^{\circ} 15^{\prime}+30^{\prime}$. The $K=1$ transitions of the asymmetrically deuterated species are split by internal rotation about the $\mathrm{C}$-Ge bond. With the assumption of a threefold sinusoidal potential, an internal barrier of $1239 \pm 25 \mathrm{cal} / \mathrm{mole}$ has been determined from these splittings. Analysis of the Stark effect of several species gives a dipole moment of $0.635 \pm 0.006$ $D$. From observed hyperfine structure the nuclear quadrupole coupling constant of $\mathrm{Ge}^{73}$ has been calculated to be $+3 \mathrm{Mc}$

Measurement of ozone in terms of its optical absorption, R. Stair, Advances in Chem. Series of the Am. Chem. Soc., No. 21, 269 (1959).

The unique absorption spectrum of ozone provides an ideal physical basis for measuring its concentration in the atmosphere even in the presence of significant quantities of other atmospheric pollutants, whether of gaseous or particulate character. Various types of optical equipment have been considered, both for measurement of the total amount of ozone and for determination of its vertical distribution and horizontal concentration. Natural sunlight furnishes a suitable and convenient light source for measuring the total amount and vertical distribution of ozone. Special sources having high radiant intensity within the spectral region of 2500 to $3600 \mathrm{~A}$, where ozone has a high optical absorption, are desired for use in measuring the horizontal concentration of ozone. The light source, whether the sun or some special source such as a mercury arc lamp, may be employed with a simple filter radiometer or with a more or less elaborate prism or grating spectroradiometer as desired. In most of the recent work at the National Bureau of Standards a double, 
quartz prism spectroradiometer has been used at Washington, D.C., Climax, Colo, Los Angeles, Calif., and Sunspot, N. Mex., in ozone studies.

Lower bounds for eigenvalues with application to the helium atom, N. W. Bazley, Proc. Nat. Acad. Sci. U.S. 45, No. 6, 850 (1959).

Let $A$ be a self-adjoint operator with domain $D$ in a Hilbert space $\mathfrak{S}_{\mathrm{j}}$. Suppose $A=A^{\prime}+\bar{A}$ where $\bar{A}$ is self-adjoint and $A^{\prime}$ is positive definite. The eigenvalue problem for $\bar{A}$, whose solution we assume known, gives rough lower bounds. If $\bar{u}_{i}$ $(i=1, \ldots, k)$ are $k$ discrete eigenvectors of $\bar{A}$ and if $P_{i}=$ $\left(A^{\prime}\right)^{-1} \bar{u}_{i}(i=1, \ldots, k)$ exist then one can substantially improve the lower bounds. The theory is applied to the helium atom operator.

Spectroscopic evidence for triatomic nitrogen in solids at very low temperature, M. Peyron, E. M. Horl, H. W. Brown, and H. P. Broida, J. Chem. Phys. 30, No. 5, 1304 (1959).

Additional wavelength, intensity and lifetime measurements of the radiation emitted from solid nitrogen containing trapped atoms have led to the interpretation that a weakly bound triatomic molecule, $\mathrm{N}_{2}-\mathrm{N}$, is an emitting species. The three lowest electronic levels of atonic nitrogen ${ }^{2} \mathrm{P},{ }^{2} \mathrm{D}$, and ${ }^{4} \mathrm{~S}$ are involved in the eight line groups which have been found. Isotopic substitution has confirmed this model. Evidence also has been found for an $\mathrm{N}_{2}-\mathrm{O}$ molecule similar to the $\mathrm{N}_{2}-\mathrm{N}$.

Thermodynamic properties of helium at low temperatures and high pressures. D. B. Mann and R. B. Stewart, NBS Tech. Note 8 (PB151367) \$1.25.

This is a compilation and correlation of the present data on the thermodynamic properties of helium below $20^{\circ} \mathrm{K}$. The existing (best) values are selected. The results are presented in the form of temperature-entropy and enthalpy-entropy diagrams. Pressures to $100 \mathrm{~atm}$, temperatures from $0^{\circ} \mathrm{K}$ to $20^{\circ} \mathrm{K}$ and specific volumes from 5 liters $/ \mathrm{kg}$ to 800 liters $/ \mathrm{kg}$ are presented.

On the perturbation of the vibrational equilibrium distribution of reactant molecules by chemical reactions, K. E. Shuler, "th Symp. (Intern.) on Combustion, London and Oxford, Aug. 28 to Sept. 3, 1958, Combustion Inst. p. 87 (Butterworths Sci. Pub., London, England, 1958).

A mathematical analysis is made of the stepwise excitation of an assembly of harmonic oscillators with an irreversible dissociation limit by a solution of the transport equations using Gottlieb polynomials. The variations in the distribution function as determined by values of the dissociation energy and the conditions for perturbation of the equilbrium Boltzmann vibrational distribution because of chemical reactions are discussed.

\section{Other NBS Publications*}

Journal of Research, Section 63B, No. 2, OctoberDecember 1959 . 75 cents.

Applications of a theorem on partitioned matrices. E. V. Haynsworth.

Capacity requirement of a mail sorting device: II. A. J. Goldman.

Analytic comparison of suggested configurations for automatic mail sorting equipment. B. K. Bender and A. J. Goldman.
New method of solution for unretarded satellite orbits. John P. Venti.

Effect of sudden water release on the reservoir free outflow hydrograph. Vujica M. Yevdjevich.

Uniform asymptotic expansions for Weber parabolic cylinder functions of large orders. F. W. J. Olver.

\section{Journal of Research, Section 63C, No. 2, October- December 1959 . 75 cents.}

Conical coaxial capacitors and their advantages. M. C. Selby.

A photoelectric followup and recording system, and its application to remote observations of the beam in high precision balances. H. A. Bowman and L. B. Macurdy.

A stroboscopic vibration analyzer. S. Edelman, R. Brooks, S. Saito, E. Jones, and E. R. Smith.

Evaluation of lens distortion by the inverse nodal slide. Francis E. Washer and Walter R. Darling.

Evaluation of lens distortion by the modified goniometric method. Francis E. Washer and Walter R. Darling.

Proposed criteria for defining load failure of beams, floors, and roof constructions during fire tests. J. V. Ryan and A. F. Robertson.

Conductive flooring for hospital operating rooms. Thomas H. Boone, Francis L. Hermach, Edgar H. MacArthur, and Rita C. McAuliff.

Measurement of the aging of rubber vulcanizates. J. Mandel, F. L. Roth, M. N. Steel, and R. D. Stiehler. (See above abstracts.)

Journal of Research, Section 63D, No. 3, November-December 1959 . 70 cents.

Radio-refractive-index climate near the ground. B. R. Bean and J. D. Horn.

Path antenna gain in an exponential atmosphere. W. J. Hartman and R. E. Wilkerson.

Effect of atmospheric horizontal inhomogeneity upon ray tracing. B. R. Bean and B. A. Cahoon.

Correlation of solar noise fluctuations in harmonically related bands. L. R. O. Storey.

A monochromatic low-latitude aurora. F. E. Roach and E. Marovich.

Pattern synthesis for slotted-cylinder antennas. James R. Wait and James Householder.

Central Radio Propagation Laboratory exponential reference atmosphere. B. R. Bean and G. D. Thayer.

Excitation mechanisms of the oxygen 5577 emission in the upper atmosphere. E. Tandberg-Hanssen and F. E. Roach. (See above abstracts).

Method for measuring local electron density from an artificial satellite. L. R. O. Storey. (See above abstracts).

Thermodynamic properties of helium at low temperatures and high pressures, D. B. Mann and R. B. Stewart, NBS Tech. Note 8 (PB151367) $\$ 1.25$ (see above abstracts). 
Transmission loss in radio propagation-II, K. A. Norton, NBS Tech. Note 12 (PB151371) \$3.00. Technical considerations leading to an optimum allocation of radio frequencies in the band 25 to 60 mc, K. A. Norton, NBS Tech. Note 13 (PB151372) \$2.50.

The role of Government research laboratories, A. V. Astin, Elec. Engr. 78, No. 7, 738 (1959).

The ampere, F. B. Silsbee, Proc. IRE 4\%, No. 5, 643 (1959).

On the theory of reflection from a wire grid parallel to an interface between homogeneous media (II), J. R. Wait, Appl. Sci. Research \%, 355 (1959).

The basis of our measuring system, A. G. McNish, Proc. IRE 4\%, No. 5, 636 (1959).

The research frontier, I. C. Schoonover, Saturday Rev. p. 58 (May 2, 1959).

Paper test data from pendulum and inertialess testers, R. J. Capott, S. B. Newman, and J. Mandel, TAPPI 42, No. 6, 480 (1959).

Convexity of the field of a linear transformation, A. J. Goldman and M. Mareus. Can. Math. Bull. 2, No. 1, 15 (1959).
Factors affecting the accuracy of distortion measurements made on the nodal slide optical bench, F. E. Washer and W. R. Darling, J. Opt. Soc. Am. 49, No. 6, 517 (1959).

IGY instruction manual. Part 1: World days and communications, A. H. Shapley, Annals of International Geophys. Year $\boldsymbol{\gamma}$, Pt. 1, 1 (Pergamon Press, New York, N.Y., 1959).

Graphical diagnosis of interlaboratory test results, W. J. Youden, Ind. Qual. Control XV, No 11, I (1959).

The calculation of the field in a homogeneous conductor with a wavy interface, J. R. Wait, Proc. IRE 4\%, 1155 (1959).

Rack for standard resistors, P. H. Lowrie, Jr., Rev. Sci. Instr. 30, No. 4, 291 (1959).

The evaluation of small color differences: Part I. Visual observations, J. C. Richmond and W. N. Harrison, Am. Ceram. Bul. 38, No. 6, 292 (1959).

* Publications for which a price is indicated (except for Technical Notes) are available only from the Superintendent of Documents, U.S. Government Printing Office, Washington 25, D.C. (foreign postage, one-fourth additional). Technical Notes are available only from the Office of Technical Services, U. S. Department of Commerce,
Washington 25, D.C. (Order by PB number.) Reprints from outside journals and the NBS Journal of Research may often be obtained directly from the authors. 